Überhaupt ist das Kulturprogramm der WLB vielfältiger als das einer Universitätsbibliothek. Zu erwähnen sind hier insbesondere die Vorträge, die von der Württembergischen Bibliotheksgesellschaft bzw. der BfZ - mit z.T. hochrenommierten Vortragenden - veranstaltet werden. Erst im vergangenen Jahr konnte mit Christopher Clark einer der weltweit bekanntesten Forscher zum Ersten Weltkrieg als Referent gewonnen werden.

Wenngleich der Erweiterungsbau kein spezifischer Schwerpunkt meines Aufenthalts an der WLB war, so war er doch - zumindest akustisch! - in (fast) allen Gesprächen präsent: Nach über zehnjähriger Planungsphase starteten die Bauarbeiten wenige Wochen vor meinem Aufenthalt an der WLB, der Rohbau soll bis Ende 2016 fertiggestellt sein und Anfang 2018 die Übergabe stattfinden. Neben einer Vielzahl von modernen Nutzer-Arbeitsplätzen soll im neuen WLB-Gebäude auch ein umfangreicher Freihand-Bereich entstehen. In diesem Zusammenhang war der an der WLB geplante Umstieg von der bisherigen hauseigenen Lesesaal-
Systematik auf die Regensburger Verbundklassifikation (RVK) für mich von großem Interesse - vor allem da auch an den Teilbibliotheken der UB Erl.-Nbg. immer wieder Umstellungen von eigenen (Instituts-)Systematiken auf die RVK durchgeführt werden.

Insgesamt war mein Aufenthalt an der WLB für mich sowohl im Umfang wie im Inhalt genau richtig: Mit vier Wochen Länge war es mir möglich, mich ausführlich in die landesbibliothekarischen Spezifika einzuarbeiten als auch im Rahmen eines eigenen Projekts tätig zu sein. Inhaltlich wurden meine bisherigen Erfahrungen an der UB Erl.-Nbg. gut ergänzt. Besonders erfreut war ich über die kollegiale Atmosphäre im Haus, die Ermöglichung von der Teilnahme an hausinternen Sitzungen und Schulungen (Verwaltungssitzung, Redaktionssitzung WLBforum, Workshops zum RVK-Umstieg) sowie die Bereitschaft der Ausbildungsleitung, auf meine Wünsche und Interessen einzugehen.

\title{
Jürgen Rohwer, 1924 - 2015
}

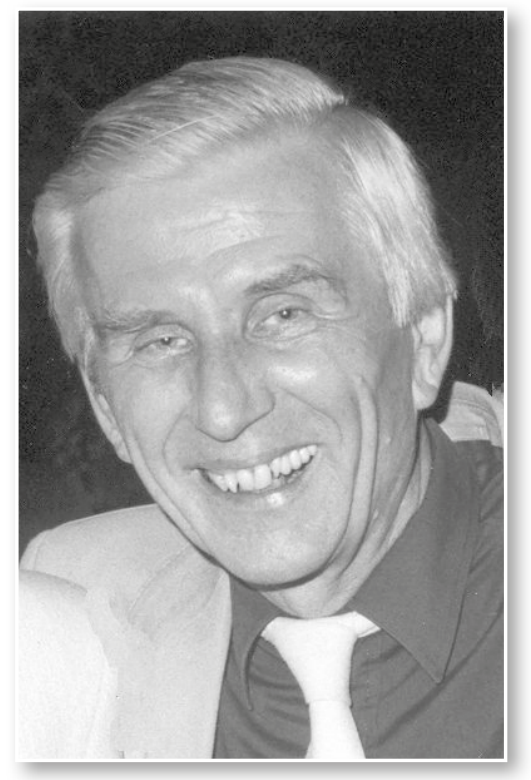

Der langjährige

Direktor der

Bibliothek für

Zeitgeschichte

(BfZ) gehörte zur

studentischen

"Generation

der 1945er" (so

der australische

Historiker Dirk

Moses). Die

erste Generation

deutscher Aka-

demiker nach

1945, die den

Krieg noch als

junge Soldaten, Flakhelfer oder als Hitlerjungen im

"Volkssturm" erlebt hatte - Jürgen Rohwer diente von 1942 - 1945 in der Kriegsmarine -, zeichnete sich durch ein "Pathos der Nüchternheit" aus - jenseits aller politischen Aufregung. Angesichts der ungeheuerlichen Verbrechen des NS-Staates und des vollständigen Bankrotts der Ideale, an die viele von innen geglaubt hatten, richteten sich die Hoffnungen dieser Generation auf einen politischen Neubeginn und eine stabile, funktionstüchtige Demokratie. Eine solche Haltung war zweifellos auch Jürgen Rohwer eigen, der nach einem Studium der Geschichte und anschließender Promotion (1954) an der Universität Hamburg zunächst als Geschäftsführer des (zeitgleich zur Gründung der Bundeswehr etablierten) Arbeitskreises für Wehrforschung in Frankfurt a. M. sowie seit 1959 als Leiter der ehemaligen Weltkriegsbücherei, nunmehr Bibliothek für Zeitgeschichte, in Stuttgart tätig war.

In beiden Funktionen bemühte er sich - ebenso erfolgreich wie nachhaltig - um eine Verwissenschaftlichung der traditionellen Kriegsgeschichte und zugleich um eine Neupositionierung der Militär- 
wissenschaften jenseits ihrer politischen Indienstnahme und Instrumentalisierung durch Reichswehr und Wehrmacht. Unter Rohwers Ägide entwickelte sich die BfZ zu einem national wie international bekannten und nachgefragten Zentrum für die Militärgeschichte des 20. Jahrhunderts. Dies gelang inm insbesondere durch eine stärkere Verknüpfung der etablierten Militärgeschichte mit der Geschichte der internationalen Beziehungen sowie der Geschichte der militärischen Technik und des Nachrichtenwesens. Hiervon künden zahlreiche gewichtige Veröffentlichungen aus seiner Feder, Dokumentationen und Sammelbände der BfZ und nicht zuletzt einige internationale Kongresse, die Rohwer gemeinsam mit seinem Historiker-Kollegen Eberhard Jäckel vom Historischen Institut der Universität Stuttgart ausrichtete. Zwei dieser Tagungen verdienen eine besondere Hervorhebung, zumal sie in mancher Hinsicht wissenschaftliches Neuland betraten bzw. weitere wichtige Forschungen anstießen: Das von Historikern, Kryptologen und Zeitzeugen bestrittene Symposium zur Funkaufklärung im Zweiten Weltkrieg (1978), zu dem die BfZ eine beachtenswerte Ausstellung beisteuerte und dabei zugleich ihr eigenes Exemplar einer RotorVerschlüsselungsmaschine („Enigma“) präsentieren konnte. Geradezu als eine Schlüsseltagung der Holocaustforschung gilt inzwischen der Stuttgarter Kongress deutscher und internationaler Experten über „Entschlußbildung und Verwirklichung" des Mordes an den europäischen Juden mit seinen ebenso engagiert wie kontrovers geführten Diskussionen (1984).

Nicht nur die Forschung profitierte von dem großen Elan und unermüdlichen Wirken des Stuttgarter Militärhistorikers, auch die Sammlungen der BfZ verzeichneten, nicht zuletzt dank Rohwers internationaler Kontakte und Präsenz, erheblichen Zugewinn. Das gilt vor allem für die Fotosammlungen des sogenannten Marinearchivs, das für Historiker wie für interessierte Laien gleichermaßen eine außerordentliche Fundgrube zur Marinegeschichte in der Neuzeit darstellt. Mit der erzwungenen Einstellung der von Jürgen Rohwer lange Jahre als Schriftleiter (und faktischem Herausgeber) geführten „Marine-Rundschau“ Mitte der 1980er Jahre kam dem Marinearchiv allerdings "nicht nur sein größter Zuträger, sondern auch sein größter Nutzer abhanden" (Thomas Weis). Auch bei dem
Erwerb und der Etablierung weiterer Sammlungen bewies Rohwer eine glückliche Hand, so etwa bei dem 1964 mit öffentlichen Mitteln erworbenen "Zarenarchiv", dem Nachlass eines Adjutanten des russischen Zaren Nikolaus II., sowie 1972 bei der Einrichtung der zunächst von der DFG geförderten „Dokumentationsstelle für unkonventionelle Literatur" (heute "Neue Soziale Bewegungen"), die u.a. das überaus heterogene Schriftgut der Studentenbewegung nach 1968 versammelte.

Die 30-jährige Amtszeit (von 1959 bis 1989) des Historikers Jürgen Rohwer war ein Glücksfall für die BfZ, deren öffentliche wie vor allem fachwissenschaftliche Wahrnehmung allzu lange von dem Bild ihrer Vorgängerin, der von Richard Franck gegründeten „Weltkriegsbücherei", bestimmt blieb. Rohwer gelang es, die BfZ allmählich aus diesem deutsch-national und militaristisch geprägten Dunstkreis herauszuführen, indem er engagiert für eine international orientierte und neuen Forschungen und Fragestellungen gegenüber aufgeschlossene Militärgeschichte warb. Ausweis der neu gewonnenen Akzeptanz waren nicht nur Rohwers Ernennung zum Honorarprofessor der Universität Stuttgart (1970) und seine Mitgliedschaften in zahlreichen nationalen wie internationalen Organisationen - so war er von 1985 bis 1990 Vizepräsident der Internationalen Kommission für Militärgeschichte -, sondern auch die Tatsache, dass die BfZ mit ihren reichen bibliothekarischen wie archivalischen Sammlungen schließlich zu einer zentralen Anlaufstelle der modernen Zeitgeschichtsforschung in Deutschland werden konnte. Dafür schulden wir Jürgen Rohwer unseren großen Dank und ein ehrenvolles Gedenken. 